Document downloaded from:

http://hdl.handle.net/10251/83355

This paper must be cited as:

Sánchez-Soriano, MÁ.; Sirci, S.; Martínez Pérez, JD.; Boria Esbert, VE. (2016). Compact Dual-Mode Substrate Integrated Waveguide Coaxial Cavity for Bandpass Filter Design. IEEE Microwave and Wireless Components Letters. 26(6):386-388. doi:10.1109/LMWC.2016.2558651.

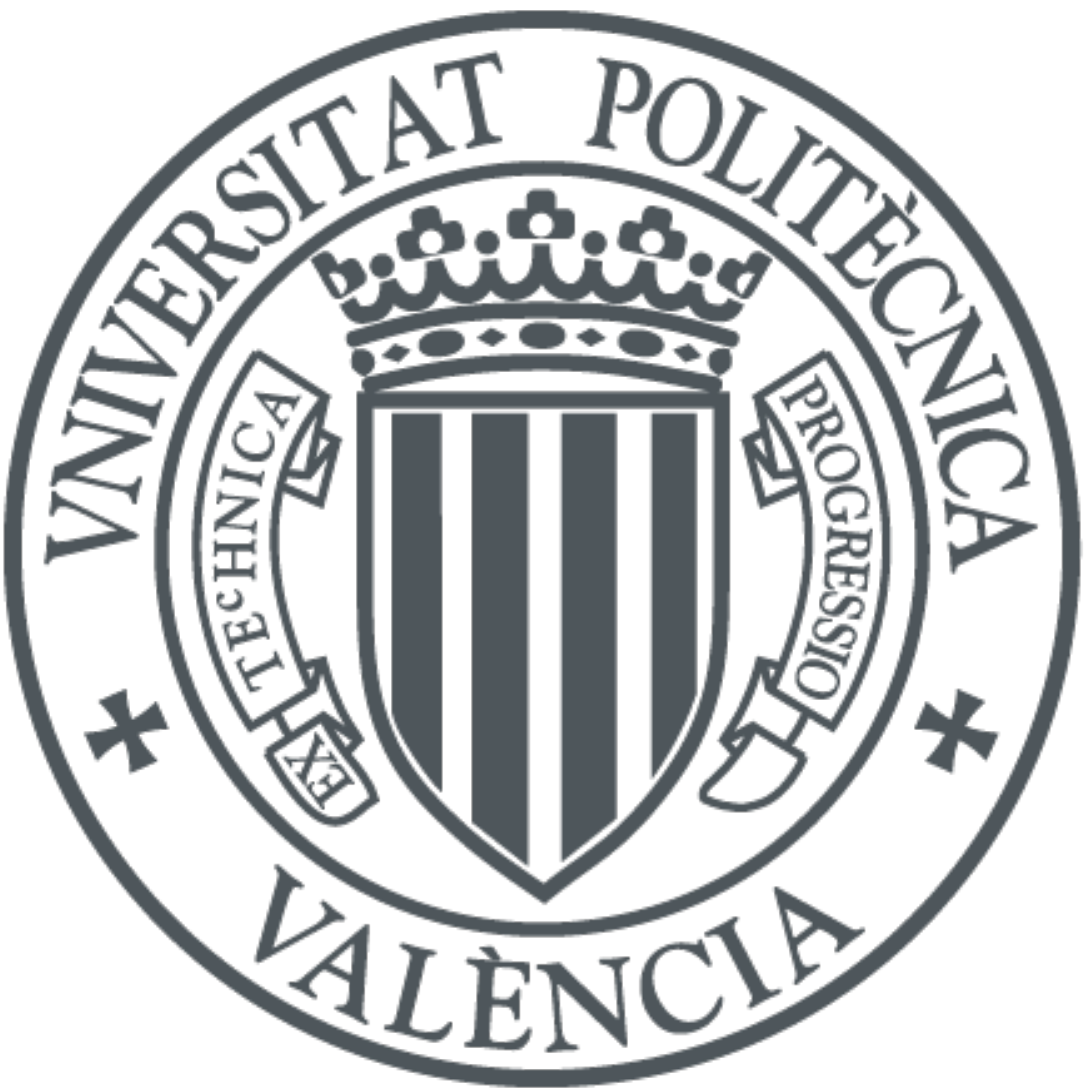

The final publication is available at

http://dx.doi.org/10.1109/LMWC.2016.2558651

Copyright Institute of Electrical and Electronics Engineers (IEEE)

Additional Information 


\title{
Compact Dual-Mode Substrate Integrated Waveguide Coaxial Cavity for Bandpass Filter Design
}

\author{
Miguel Á. Sánchez-Soriano, Member, IEEE, Stefano Sirci, Student Member, IEEE, \\ J. D. Martínez, Member, IEEE, and Vicente E. Boria, Senior Member, IEEE
}

\begin{abstract}
This letter proposes a new compact filtering building block. It consists of two via holes embedded into a substrate integrated waveguide (SIW) cavity connected to capacitive metal patches at the top layer. This topology provides two coaxial modes performing a doublet filtering configuration. The proposed dualmode SIW coaxial cavity is studied in detail and guidelines for the filter design are given. As will be shown, the proposed building block presents a high degree of design flexibility, which allows for the design of multiple kind of bandpass filter (BPF) responses, including both narrow- and wide-band BPFs along with transmission zero generation. As a verification, several filters are designed and implemented at $8 \mathrm{GHz}$.
\end{abstract}

Index Terms-Bandpass filter (BPF), coaxial line, dual-mode, substrate integrated waveguide (SIW), transmission zero (TZ).

\section{INTRODUCTION}

$\mathbf{T}$ HE hybrid technology substrate integrated waveguide (SIW) has demonstrated in the last years to be a very attractive solution for managing propagation, power division and/or filtering of signals in microwave/mm-wave systems. This is mainly due to its low cost, easy integration with classical planar technologies, such as microstrip or coplanar, and its relatively high $Q$-factor [1]. With respect to the filtering application, the emergent microwave applications demand advanced filtering responses with several transmission zeros (TZs) in their responses to avoid, for example, co-channel interferences, along with a very compact circuit size. SIW components, for not very high frequencies (i.e., lower than $10 \mathrm{GHz}$ ), usually present a big occupation area, mainly in comparison to their microstrip counterparts. In order to face this issue, several solutions/approaches have been proposed for SIW compactness, such as the folded SIW [2], half-mode SIW [3] or more recently the coaxial combline SIW [4], where a via hole is embedded into the center of the SIW cavity providing a TEM coaxial mode.

In this letter, a filtering building block consisting of a dualmode SIW coaxial cavity is proposed. Such a building block

Manuscript received January 14, 2016; accepted March 14, 2016. This work has been supported in part by MINECO (Spanish Government) under projects TEC2013-47037-C5-1-R and TEC2013-48036-C3-3-R, and by a "Juan de la Cierva" Research fellowship.

M. Á. Sánchez-Soriano is with the Department of Physics, Systems Engineering and Signal's Theory, University of Alicante, 03690 Alicante, Spain (e-mail: m.sanchez.soriano@ieee.org).

S. Sirci and V. E. Boria are with the iTEAM, Universitat Politècnica de València, 46022 Valencia, Spain.

J. D. Martínez is with the $\mathrm{I} 3 \mathrm{M}$, Universitat Politècnica de València, 46022 Valencia, Spain.

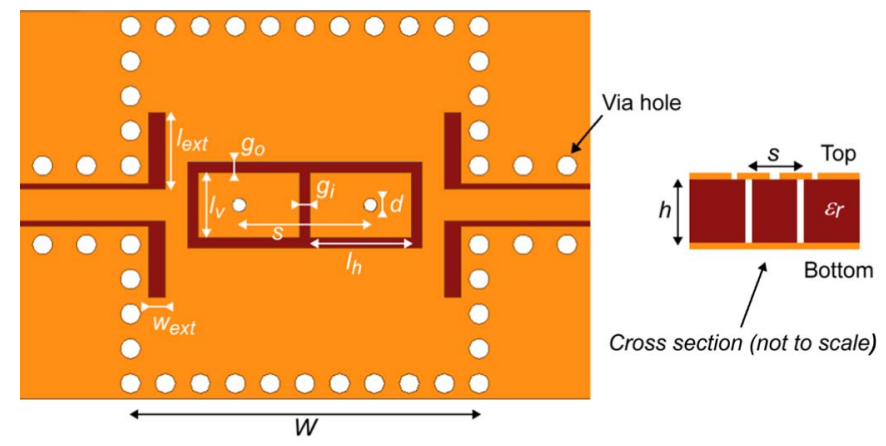

(a)

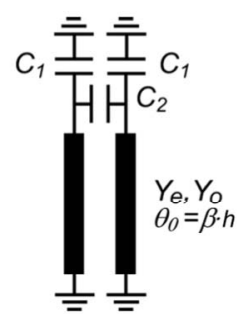

(b)

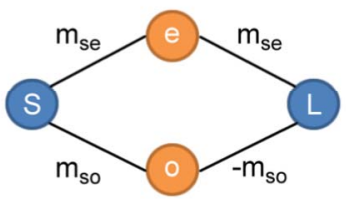

(c)
Fig. 1. (a) Layout of the proposed dual mode SIW coaxial building block. (b) Equivalent transmission line model. (c) Routing coupling path.

provides a high degree of miniaturization along with a high filter design flexibility. The proposed building block also generates a TZ, which can be placed either below or above the passband, as desired for the filter designer. Several bandpass filters (BPFs) presenting narrow- and wide-band responses, centered at $8 \mathrm{GHz}$ and based on the proposed building block are designed and implemented, thus demonstrating the aforementioned advantageous features.

\section{Theoretical AnAlysis of the PRoposed BuILDING BLOCK}

Fig. 1(a) shows the layout of the proposed dual-mode SIW coaxial cavity. It is formed by a SIW cavity where two via holes are symmetrically inserted with respect to the cavity center. Each via hole with diameter $d$ is connected to the circuit ground at the bottom and to a capacitive patch at the top. This configuration can be modeled as two TEM-mode combline resonators of length $h$ which are coupled to each other by means of a distributed coupling, which is defined mainly by the separation between via holes $s$, and by a lumped capacitance associated to the capacitive coupling between metallic patches $C_{2}$. Fig. 1(b) shows the equivalent transmission line model describing the proposed building block. The outer perimeter of each patch $2 l_{h}+l_{v}$ and the gap $g_{o}$ determine the capacitance 
value $C_{1}$, whereas $C_{2}$ depends on the length $l_{v}$ and the inside gap $g_{i}$. Their values can be computed by means of a quasistatic simulation or by using the approach of [5]. Note that the $\mathrm{Cu}$ base thickness $t$ also plays an important role in both capacitance values. This equivalent circuit, due to its symmetry, can be analyzed by means of an even-odd mode analysis. The two transcendental equations which provide the two resonant frequencies of the proposed dual-mode cavity are found as

$$
\omega_{e} C_{1}=\frac{Y_{e}}{\tan \frac{\omega_{e}}{v} h} ; \quad \omega_{o}\left(C_{1}+2 C_{2}\right)=\frac{Y_{o}}{\tan \frac{\omega_{o}}{v} h}
$$

where $\omega_{e}$ and $\omega_{o}$ and, $Y_{e}$ and $Y_{o}$ are the angular resonant frequencies and characteristic admittances of the even and odd mode, respectively, and $v$ is the propagation speed. $Y_{e}$ and $Y_{o}$ can be computed numerically by using static methods such as conformal transformation or Green's function [6], [7]. Their values depend on the ratio $d / W$ (where $W$ is the cavity width), $s$ and the substrate permittivity $\epsilon_{r}$. Obviously, as $s$ increases, the ratio $Y_{o} / Y_{e}$ approaches to 1 and no distributed coupling appears between lines. For small resonator electrical lengths, as usually happens in SIW combline resonators, one can find a closed expression for the ratio between the two resonant frequencies, which facilitates the filter design:

$$
\frac{f_{o}}{f_{e}}=\sqrt{\frac{C_{1}}{C_{1}+2 C_{2}} \frac{Y_{o}}{Y_{e}}}
$$

As deduced from (2), it is possible to synthesize the required $f_{o} / f_{e}$ by controlling both the coupling between lines $\left(Y_{o} / Y_{e}\right)$ and $C_{1}$ and $C_{2}$. The ratio $f_{o} / f_{e}$ defines the passband bandwidth of the filter response, indeed, this topology allows us the design of both narrowband and wideband filters by just choosing properly $f_{o} / f_{e}$. Other characteristics such as the position of the generated TZ, can also be easily controlled with this topology as will be shown next. It should be noted that $f_{o} / f_{e}$ can be set to a value higher or lower than 1 , depending on the desired filter performance. Therefore, the proposed building block provides a high degree of flexibility.

For the filter design purpose, the doublet filtering configuration shown in Fig. 1(c) perfectly models the proposed building block. This configuration provides two poles and one finite TZ [8]. The TZ is placed at the normalized frequency

$$
\Omega_{\mathrm{TZ}}=\frac{m_{\mathrm{oo}} m_{\mathrm{se}}^{2}-m_{\mathrm{ee}} m_{\mathrm{so}}^{2}}{m_{\mathrm{so}}^{2}-m_{\mathrm{se}}^{2}}
$$

where $m_{\mathrm{ee}}$ and $m_{\mathrm{oo}}$ are the normalized even and odd mode frequencies, and $m_{\mathrm{se}}$ and $m_{\mathrm{so}}$ are the normalized couplings between the input and the even- and odd-modes, respectively. Thus, by properly choosing the design parameters, the TZ can be placed at frequencies lower or higher than the filter passband, as required for the application. It should be noted that there is not coupling between modes in the proposed topology, independently of the value of coupling between lines. The parameters $m_{\text {se }}$ and $m_{\text {so }}$ are controlled by the penetration of the coplanar feeding line into the cavity through $l_{\text {ext }}$ and $w_{\text {ext }}$. For the proposed filtering building block, $m_{\text {se }}>m_{\text {so }}$ for any geometrical parameter, which means that the input (output) port is coupled more strongly to the even mode than to the odd mode.
TABLE I

Filter DESIGN PARAMETERS

\begin{tabular}{l|c|c|c|c}
\hline \hline & $Y_{e}(\mathrm{mS})$ & $Y_{o}(\mathrm{mS})$ & $C_{1}(\mathrm{fF})$ & $C_{2}(\mathrm{fF})$ \\
\hline Filter WB-I & 7.30 & 13.89 & 325 & 74 \\
Fiter WB-II & 9.46 & 11.15 & 344 & 73 \\
Filter NB & 9.40 & 11.16 & 340 & 53 \\
\hline
\end{tabular}

TABLE II

FILTER DIMENSIONS (UNITS IN mm)

\begin{tabular}{l|c|c|c|c|c|c|c}
\hline \hline & $l_{h}$ & $l_{v}$ & $s$ & $g_{i}$ & $g_{o}$ & $l_{\text {ext }}$ & $w_{\text {ext }}$ \\
\hline Filter WB-I & 1.8 & 2.2 & 2.95 & 0.15 & 0.15 & 3.5 & 0.8 \\
Fiter WB-II & 3.0 & 2.0 & 5.15 & 0.15 & 0.35 & 3.5 & 0.8 \\
Filter NB & 3.0 & 2.0 & 5.3 & 0.30 & 0.30 & 2.3 & 0.5 \\
\hline
\end{tabular}

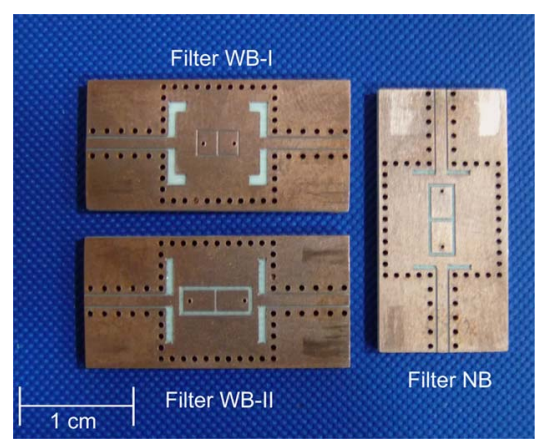

Fig. 2. Photographs of the three implemented filters.

\section{EXPERIMENTAL VALIDATION}

In order to validate the proposed topology, several second order BPFs presenting narrow and wideband responses have been designed and fabricated by using a standard PCB prototyping process. The filters have been implemented on RO4003C Rogers substrate, whose characteristics are: $\epsilon_{r}=3.55$, $h=1.52 \mathrm{~mm}, t=17 \mu \mathrm{m}$, and loss tangent $\tan \delta=0.003$. All filters have been designed at a center frequency $f_{0}=8 \mathrm{GHz}$. For all designs $d=0.4 \mathrm{~mm}$ and $W=10.6 \mathrm{~mm}$. Those values are chosen as a trade-off between $Q$-factor and compactness [4]. The unloaded $Q$-factor of the proposed dual-mode cavity is around 220, extracted from HFSS simulations.

\section{A. Wideband BPF Examples}

In this first example, a wideband BPF (named WB-I) with a fractional bandwidth $\mathrm{BW}_{3 \mathrm{~dB}}=20 \%$ is designed and implemented. The TZ is designed to be above the passband. The filter design parameters and dimensions are given in Tables I and II, whereas the photographs of the implemented filters are shown in Fig. 2. The filter presents a very compact size of $10.6 \times$ $10.6 \mathrm{~mm}^{2}$ (i.e., $0.42 \times 0.42 \lambda_{g}^{2}$, where $\lambda_{g}$ is the guided wavelength of the CPW feeding line). A conventional 2nd order SIW filter based on rectangular cavities coupled by means of iris windows would occupy a size around 4 times bigger without presenting any $\mathrm{TZ}$ in the response. Fig. 3 plots the theoretical, simulated (by using HFSS) and measured responses, where a good agreement is observed among all of them. The in-band return and insertion losses are in all responses better than $15 \mathrm{~dB}$ and $0.7 \mathrm{~dB}$, respectively. The filter presents a high selectivity in the higher band part due to the generated TZ. The achieved wide fractional bandwidth would be very difficult to be obtained by using conventional SIW filter configurations, or even with the recently proposed SIW coaxial filters [4]. 


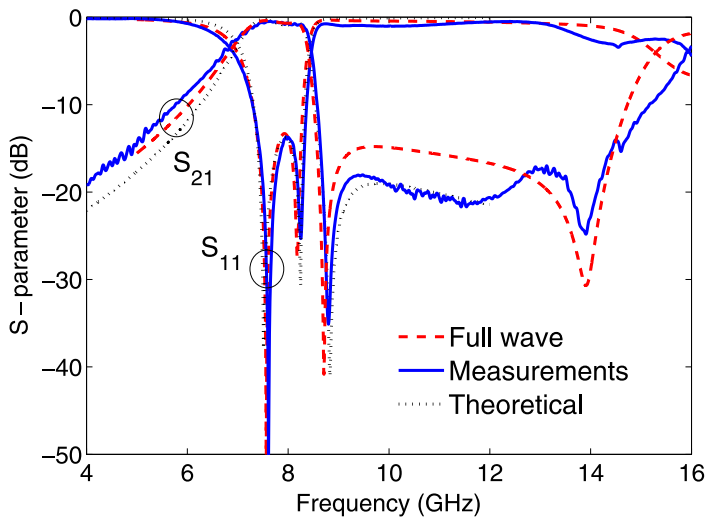

Fig. 3. Theoretical, full-wave simulated and measured responses of the implemented Filter WB-I. For the theoretical response: $m_{\mathrm{se}}=0.62, m_{\mathrm{so}}=0.35$, $m_{\mathrm{ee}}=0.70$ and $m_{\mathrm{oo}}=-0.38$ with $\mathrm{BW}_{3 \mathrm{~dB}}=20 \%$.

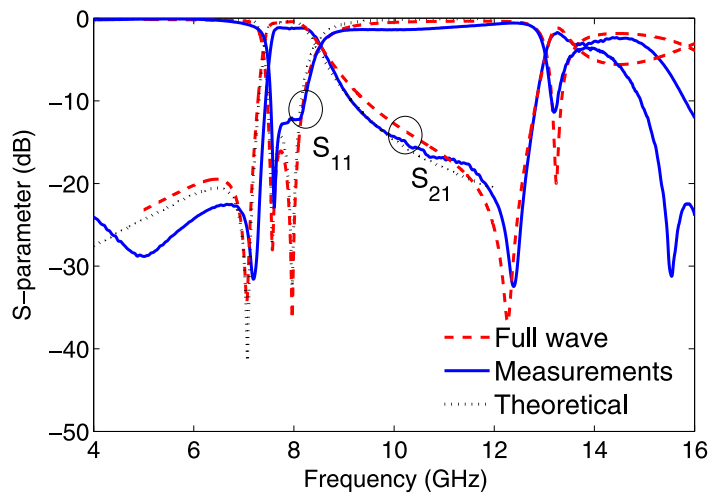

Fig. 4. Theoretical, full-wave simulated and measured responses of the implemented Filter WB-II. For the theoretical response: $m_{\mathrm{se}}=0.63, m_{\mathrm{so}}=0.39$, $m_{\mathrm{ee}}=-0.18$ and $m_{\mathrm{oo}}=1.04$ with $\mathrm{BW}_{3 \mathrm{~dB}}=14 \%$.

Another wideband BPF (WB-II) with $\mathrm{BW}_{3 \mathrm{~dB}}=14 \%$ is designed and implemented in order to demonstrate the high design flexibility of the proposed building block. In this case, the TZ is placed below the passband and the wideband response is obtained with a very low distributed coupling between lines, i.e., $Y_{o} / Y_{e} \approx 1$, as Table I depicts. Fig. 4 shows the simulated and measured responses. Again, a good agreement between all responses is found, whereas the size remains the same. The stopband of the filter WB-I is spurious free up to $16 \mathrm{GHz}$, where the SIW cavity $\mathrm{TE}_{101}$ mode is excited. In filter WB-II the first spurious band appears at a lower frequency (around $13 \mathrm{GHz}$ ), due to the excitation of the higher-order even mode of the proposed dual-mode cavity.

\section{B. Narrowband BPF Example}

In this last example, a narrowband filter, named NB, with $\mathrm{BW}_{3 \mathrm{~dB}}=5 \%$ and with the $\mathrm{TZ}$ placed below the passband is implemented. Fig. 5 depicts the simulated and measured results. The measured insertion loss at $f_{0}$ is $1.7 \mathrm{~dB}$, whereas the inband return loss is better than $11 \mathrm{~dB}$. Again, the versatility of the proposed building block is fully demonstrated.

Finally, Table III provides a brief comparison of these prototypes with other compact SIW BPFs recently reported. The compact size and the TZ generation of the proposed topologies must be remarked.

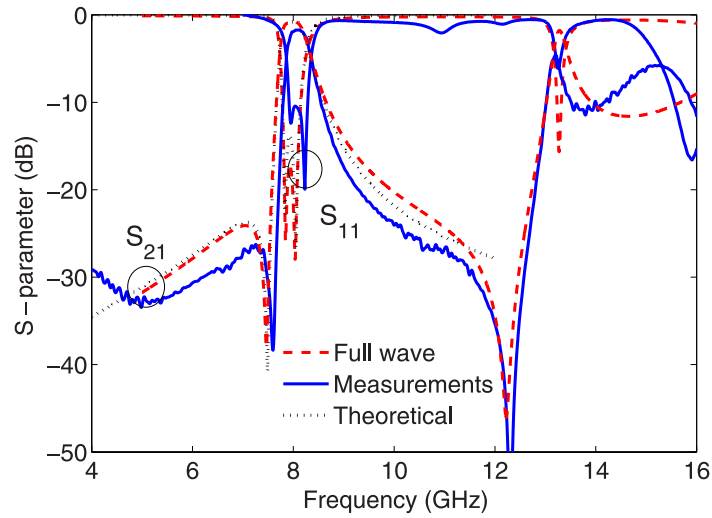

Fig. 5. Theoretical, full-wave simulated and measured responses of the implemented Filter NB. For the theoretical response: $m_{\mathrm{se}}=0.73, m_{\mathrm{so}}=0.49$, $m_{\mathrm{ee}}=-0.61$ and $m_{\mathrm{oo}}=1.21$ with $\mathrm{BW}_{3 \mathrm{~dB}}=5 \%$.

TABLE III

COMPARISON WITH OTHER COMPACT SIW FILTERS

\begin{tabular}{c|c|c|c|c|c}
\hline \hline & $B W_{3 \mathrm{~dB}}(\%)$ & $f_{0}(\mathrm{GHz})$ & $\mathrm{N}$ & $\mathrm{TZs}^{\mathrm{a}}$ & Eq. Cavity Size \\
\hline$[2]$ & 5 & 10 & 4 & 0 & $0.27 \lambda_{g}^{2}$ \\
{$[3]$} & 22 & 7.8 & 3 & 0 & $0.22 \lambda_{g}^{2}$ \\
{$[4]$} & 6 & 9.8 & 3 & 0 & $0.18 \lambda_{g}^{2}$ \\
This work: & & & & & \\
WB-I & 20 & 8.0 & 2 & 1 & $0.09 \lambda_{g}^{2}$ \\
WB-II & 14 & 8.0 & 2 & 1 & $0.09 \lambda_{g}^{2}$ \\
NB & 5 & 8.0 & 2 & 1 & $0.09 \lambda_{g}^{2}$ \\
\hline
\end{tabular}

\section{CONCLUSION}

In this letter, a dual-mode SIW coaxial cavity has been presented. The proposed dual-mode building block has been analyzed in detail, and a design strategy has been given for the filter design. Along with its very compact size, its high design flexibility should be highlighted, as it has been demonstrated with the design of both narrow and wide-band BPFs presenting TZs placed as required for the particular application. Several proofof-concept filters have been successfully constructed and tested based on the proposed filtering topology. As future work, the proposed building block may be used for the design of higherdegree filters or even for filter tunability/reconfigurability.

\section{REFERENCES}

[1] X.-P. Chen and K. Wu, "Substrate integrated waveguide filters: Practical aspects and design considerations," IEEE Microw. Mag., vol. 15, pp. 75-83, Nov. 2014.

[2] N. Grigoropoulos, B. Sanz-Izquierdo, and P. Young, "Substrate integrated folded waveguides (SIFW) and filters," IEEE Microw. Wireless Compon. Lett., vol. 15, pp. 829-831, Dec. 2005.

[3] Y. Wang, W. Hong, Y. Dong, B. Liu, H. J. Tang, J. Chen, X. Yin, and K. Wu, "Half mode substrate integrated waveguide (hmsiw) bandpass filter," IEEE Microw. Wireless Compon. Lett., vol. 17, pp. 265-267, Apr. 2007.

[4] J. D. Martinez, S. Sirci, M. Taroncher, and V. E. Boria, "Compact CPWfed combline filter in substrate integrated waveguide technology," IEEE Microw. Wireless Compon. Lett., vol. 22, pp. 7-9, Jan. 2012.

[5] H. Lee and H. Eom, "Potential distributions through an annular aperture with a floating inner conductor," IEEE Trans. Microw. Theory Tech., vol. 47, pp. 372-374, Mar. 1999.

[6] R. Levy, "Conformal transformations combined with numerical techniques, with applications to coupled-bar problems," IEEE Trans. Microw. Theory Tech., vol. 28, pp. 369-375, Apr. 1980.

[7] A. Borji, S. Safavi-Naeini, and S. Chaudhuri, "TEM properties of shielded homogeneous multiconductor transmission lines with pec and pme walls," in Proc. IEEE MTT-S Int. Dig. , May 2001, vol. 2, pp. 731-734.

[8] U. Rosenberg and S. Amari, "Novel coupling schemes for microwave resonator filters," IEEE Trans. Microw. Theory Tech., vol. 50, pp. 2896-2902, Dec. 2002 